\title{
Comparação funcional entre membro dominante e não dominante em atletas com dor inguinal*
}

\section{Comparison between the Dominant and Non-dominant Sides of Athletes with Groin Pain}

\author{
Guilherme Guadagnini Falótico ${ }^{1}$ André Orlandi Bento ${ }^{1} \quad$ Luana Tossolini Goulart $^{1} \quad$ Leonardo Zafalon ${ }^{1}$ \\ Gustavo Gonçalves Arliani ${ }^{1}$ Móises Cohen ${ }^{1}$ \\ ${ }^{1}$ Centro de Traumatologia do Esporte, Universidade Federal de São Paulo, \\ São Paulo, SP, Brasil \\ Rev Bras Ortop 2020;55(2):203-207. \\ Endereço para correspondência Guilherme Guadagnini Falótico, \\ Master, Centro de Traumatologia do Esporte, Universidade Federal de \\ São Paulo, 783 Borges Lagoa Street, 5th floor. Vila Clementino, \\ São Paulo/SP -04038-003, Brasil (e-mail: ggfalotico@yahoo.com.br).
}

\section{Resumo \\ Palavras-chave \\ - atletas \\ - dor \\ - quadril \\ - lesões no quadril}

Objetivo Comparar a diferença do arco de movimento (ADM) entre o quadril com lesão e o não lesionado de atletas com as principais patologias femoroacetabulares. Além disso, analisar a diferença da ADM do quadril em cada patologia considerada. Métodos Os participantes do presente estudo foram 75 atletas de diversas modalidades esportivas, sendo 56 mulheres e 19 homens, com idades entre 20 e 46 anos, os quais tinham diagnóstico de lesão do quadril. Esses indivíduos foram subdivididos em três grupos de acordo com as patologias (impacto femoroacetabular ou lesão labral, pubalgia ou lesão dos adutores e síndrome trocantérica) e as características de cada quadril foram analisadas.

Resultados Um total de 150 quadris (direito e esquerdo) foram mensurados para a presente análise. Comparou-se o ADM do quadril lesado e do quadril saudável de cada atleta e não foram encontradas diferenças estatísticas. Da mesma forma, não houve diferença significativa entre a ADM do quadril entre as principais injúrias.

Conclusão O presente estudo não encontrou diferenças no arco de movimento entre o quadril lesionado e o contralateral, bem como não houve diferença na amplitude dos movimentos entre as patologias femoroacetabulares analisadas.

Objective To compare the difference in range of motion (ROM) between the dominant and nondominant hips of the athletes and to correlate the results with groin pain, as well as to compare the differences in ROM among the main hip injuries. Methods The participants included 75 athletes, 56 males and 19 females, aged between 20 and 46 years old, who were diagnosed with hip injury. These individuals were subdivided according to the pathologies (femoroacetabular impact or labral lesion, adductor and pubic lesions and trochanteric syndrome) and characteristics of each hip were analyzed.

Trabalho desenvolvido no Centro de Traumatologia do Esporte,

Universidade Federal de São Paulo, São Paulo, SP, Brasil.

recebido

30 de Novembro de 2018

aceito

05 de Fevereiro de 2019
DOI https://doi.org/

10.1055/s-0039-3400521. ISSN 0102-3616.
Copyright $\odot 2020$ by Sociedade Brasileira License terms de Ortopedia e Traumatologia. Published by Thieme Revinter Publicações Ltda, Rio de Janeiro, Brazil 
Keywords

- athletes

- pain

- hip

- hip injuries
Results A total of 150 hips (right and left) were measured for the present analysis. When comparing the ROM of the injured hip with the healthy hip, no statistically significant differences were found. There were also no significant differences between the amplitudes of hip movement when the main pathologies were compared.

Conclusion The present study did not find differences in ROM rotation between the various pathologies of the hip.

\section{Introdução}

O quadril é uma articulação de anatomia e biomecânica complexa, o que dificulta a identificação de fatores relacionados às lesões e à realização de um diagnóstico preciso. $^{1,2}$

Lesões musculoesqueléticas relacionadas ao esporte são mais comuns em atletas participantes de modalidades que envolvem deslocamentos alterolaterais, mudanças de direção repentinas, acelerações e desacelerações bruscas e chutes. $^{3}$ Sintomas podem variar de episódios intermitentes de desconforto, dor crônica severa, podendo até determinar o fim da carreira de alguns atletas. ${ }^{4}$

Entre as patologias mais presentes no quadril do atleta estão: lesão dos músculos adutores, com 10 a 18\%, ${ }^{5,6}$ pubalgia, que representa de 2 a $5 \%,{ }^{7,8}$ por volta de $95 \%$ apresentam sinais de impacto femoroacetabular (IFA), ${ }^{9}$ e síndrome trocantérica corresponde a $2,5 \%{ }^{10}$

Diversos fatores de risco têm sido listados para essas lesões, entre eles, uma possível assimetria da rotação do quadril. Estudos têm mostrado que indivíduos normais apresentam simetria das amplitudes de rotação interna $e$ externa do quadril ${ }^{11}$ e pacientes que se recuperam de dores no quadril têm maior amplitude de movimento (ADM) do que aqueles que se mantém sintomáticos. ${ }^{12}$ Com isso, um crescente número de trabalhos sugere que assimetria e limitação da ADM da articulação femoroacetabular são fatores de risco para dor crônica na região inguinal em atletas. $^{13}$

Uma ADM limitada da articulação coxofemoral tem sido notada em atletas diagnosticados com osteíte púbica e com fratura por estresse do osso púbico, além de sugerir uma relação com lesões dos músculos adutores. ${ }^{14}$ Outros estudos mostram que indivíduos com rotação assimétrica do quadril apresentavam também fraqueza desses músculos, geralmente quando $>15^{\circ}$ de diferença entre a rotação externa e interna de um determinado lado. Essa limitação de movimento leva a uma provável perda de força e associada a atividades vigorosas que exigem rotação e deslocamentos laterais bruscos, contato físico e chutes, pode contribuir para 0 aparecimento de sintomas. ${ }^{15}$

Diante disso, o objetivo principal do presente estudo foi comparar a ADM da articulação coxofemoral em atletas diagnosticados com pubalgia, síndrome trocantérica ou IFA e analisar a restrição de mobilidade gerada por cada patologia. Além disso, o outro objetivo foi comparar o ADM entre o quadril lesionado e o não lesionado de cada atleta.

\section{Materiais e Métodos}

Foram analisados os prontuários dos pacientes que passaram pelo Ambulatório do Quadril do Centro de Traumatologia do Esporte da nossa instituição, no período entre os anos de 2008 e 2016. Os critérios de inclusão utilizados foram indivíduos com diagnóstico médico de lesões específicas no quadril (IFA, lesão labral, pubalgia, lesão de adutores e síndrome trocantérica) realizado por meio do exame clínico associado ou não, conforme necessidade, a exames complementares (radiografia, ultrassonografia ou ressonância nuclear magnética) e que foram avaliados pela equipe de fisioterapia da nossa instituição.

Foram excluídos aqueles com dores referidas da coluna lombar e articulação sacroilíaca, casos com fraturas prévias e osteoartrose, bem como os indivíduos que não apresentavam avaliação fisioterapêutica ou diagnóstico médico concluído. As informações da anamnese, do exame físico e de questionários foram tabeladas e analisadas a fim de encontrar dados que mantivessem relação com algum tipo de lesão específica. Essas informações continham: idade, gênero, diagnóstico médico, mecanismo de lesão (traumático ou atraumático), amplitudes de rotação interna e externa, e o resultado do questionário Harris Hip Score.

Foram avaliados 133 prontuários, dos quais 44 foram excluídos do trabalho por não apresentarem avaliação fisioterapêutica ou diagnóstico médico concluído. Dos 89 restantes, 14 apresentaram diagnósticos que não se encaixavam com a proposta do trabalho, como osteoartrose, fratura de fêmur e lesões musculares (reto femoral, isquiotibiais), e por isso foram excluídos. Restaram 75 indivíduos a serem estudados, sendo 56 do sexo masculino e 19 do sexo feminino, com média de idade de 33.16 anos $( \pm 13.01)$. Destes, 22 tinham diagnóstico de IFA ou lesão labral (GRUPO 1), 36 compuseram o grupo pubalgia/lesão dos adutores (GRUPO 2) e 17 foram alocados no grupo síndrome trocantérica (GRUPO 3).

Quanto à aferição das medidas da articulação femoroacetabular, foi utilizado um goniômetro de $20 \mathrm{~cm}$ (Carci, São Paulo, SP, Brasil). $O$ atleta foi posicionado sentado na borda de uma maca, com a coxa apoiada, fletida a $90^{\circ}$, e os joelhos também fletidos a $90^{\circ}$. 0 braço estacionário do goniômetro foi alinhado perpendicularmente ao solo e o braço móvel foi alinhado com o eixo da tibia do atleta. 0 quadril do jogador foi movido passivamente em rotação interna e depois em rotação externa até detectar a sensação final da cápsula articular. Quanto à medida da flexão do quadril, o atleta estava em decúbito dorsal, o braço fixo do goniômetro, paralelo ao solo, na linha média axilar, e o braço móvel 
alinhado com o eixo femoral. Já na medida da extensão, o atleta estava em decúbito ventral, o braço estacionário e móvel do goniômetro posicionado da mesma forma que para a medida da flexão. Os testes foram realizados por três fisioterapeutas diferentes, de forma aleatória, conforme o dia da consulta do atleta e a escala de trabalho do profissional. Ainda sobre as medidas, essas foram realizadas três vezes para cada movimento de cada quadril e foi feita, por fim, uma média entre os três valores obtidos para alcançar o valor final utilizado.

Em relação às modalidades realizadas pelos 75 atletas em estudo, 30 praticavam futebol, 9 faziam atletismo, 8 lutavam karatê, 7 jogavam handebol, 6 eram nadadores, 4 eram bailarinas e 4 eram ciclistas.

\section{Análise Estatística}

Inicialmente, foi utilizada a análise descritiva para medidas antropométricas. $\mathrm{O}$ teste de Kolmogorov-Smirnov foi empregado para verificar a normalidade dos dados. Após essa etapa, lançou-se mão da análise de variância (ANOVA, na sigla em inglês) para comparar a amplitude de movimento do quadril entre os grupos, sendo que para análise intragrupos foi utilizado o teste de post hoc de Tukey. $O$ teste T pareado foi utilizado para verificar as diferenças entre o membro lesionado e não lesionado dentro de cada grupo. O programa utilizado foi o SPSS Statistics for Windows,versão 17 (SPSS Inc., Chicago, IL, EUA) e foi aceito o nível de significância de 5\% $(p \geq 0,05)$.

\section{Resultados}

Não foi encontrada diferença significativa entre os grupos quanto à avaliação do ADM do quadril lesionado e não lesionado e do nível de funcionalidade através do Harris Hip Score $(p>0,05)$ ( - Tabela 1). Além disso, na avaliação de cada atleta, não se constatou diferença da amplitude movimento da articulação dolorosa e a contralateral. Os valores obtidos estão incluídos na - Tabela 1.

\section{Discussão}

O presente estudo não encontrou diferença entre a ADM do quadril entre os grupos, nem entre o membro lesionado e não lesionado dentro de cada grupo, o que era esperado de acordo com alguns trabalhos encontrados na literatura. Estudos como o de Ibrahim et al ${ }^{12}$ mostraram que a limitação de ADM pode estar associada a lesões no quadril, apesar do mecanismo envolvido não estar claramente descrito. Fricker et al $^{16}$ descreveram um mecanismo no qual a articulação femoroacetabular passa por uma inflamação e cicatrização como parte de uma síndrome de overuse, semelhante ao ombro congelado desencadeado por uma lesão do manguito rotador. Sendo por privação vascular de estruturas pélvicas ou de focos de inflamação no quadril, a rigidez resultante é uma manifestação do espasmo reflexo dos músculos rotadores, flexores e adutores do quadril. Essas alterações podem explicar porque após uma lesão de adutores, há piora na limitação de rotação, dor à mobilização do quadril e fraqueza dos adutores. Quando a fase aguda cessa, a rotação do quadril retorna à sua amplitude pré-lesão. 0 mecanismo para isso, apesar de não inteiramente claro, inclui reabilitação agressiva, repouso e remoção dos agentes álgicos. A manutenção deste ADM reduzido, seja por uma reabilitação inadequada ou pela ausência de tratamento, pode caracterizar um fator de risco para a lesão de adutor. Alguns trabalhos mostram que os músculos adutores agem como rotadores internos do quadril quando em extensão. ${ }^{17}$ Essa contração dos adutores contra uma cápsula rígida pode ser outra possível causa para lesão destes músculos. ${ }^{12}$ Williams $^{18}$ descreveu que atividades esportivas como

Tabela 1 Mensuração do arco de movimento do quadril de atletas

\begin{tabular}{|l|l|l|l|l|l|l|l|}
\hline & GRUPO 1 & $\begin{array}{l}\text { valor-p } \\
\text { grupo 1 }\end{array}$ & GRUPO 2 & $\begin{array}{l}\text { valor-p } \\
\text { grupo 2 }\end{array}$ & GRUPO 3 & $\begin{array}{l}\text { valor-p } \\
\text { grupo 3 }\end{array}$ & $\begin{array}{l}\text { valor-p } \\
\text { entre os } \\
\text { grupos } \\
\text { (ANOVA) }\end{array}$ \\
\hline RI LL & $34.9545( \pm 13.86835)$ & 0.341 & $\begin{array}{l}36.2222 \\
( \pm 10.02030)\end{array}$ & 0.335 & $34.1765( \pm 12.12041)$ & 0.953 & 0.822 \\
\hline RI LNS & $36.3636( \pm 11.23577)$ & & $35.0833( \pm 9.06918)$ & & $34.0588( \pm 10.12096)$ & & 0.770 \\
\hline RE LL & $40.9091( \pm 14.75162)$ & 0.300 & $40.1111( \pm 12.37689)$ & 0.981 & $36.7647( \pm 12.77463)$ & 0.579 & 0.592 \\
\hline RE LNL & $42.8636( \pm 13.29982)$ & & $40.0833( \pm 13.13746)$ & & $35.7059( \pm 11.79388)$ & & 0.234 \\
\hline $\begin{array}{l}\text { ADM } \\
\text { TOTAL } \\
\text { LL }\end{array}$ & $75.8636( \pm 24.90036)$ & 0.132 & $76.3333( \pm 16.91660)$ & 0.432 & $70.9412( \pm 21.04302)$ & 0.640 & 0.652 \\
\hline $\begin{array}{l}\text { ADM } \\
\text { TOTAL } \\
\text { LNL }\end{array}$ & $79.2273( \pm 20.28119)$ & & $75.1667( \pm 17.72569)$ & & $69.7647( \pm 19.26632)$ & & 0.305 \\
\hline $\begin{array}{l}\text { HARRIS } \\
\text { HIP } \\
\text { SCORE }\end{array}$ & 70.5909 & 0.454 & 71.4722 & & 0.454 & 67.5294 & \\
\hline
\end{tabular}

Abbreviations: ADM, amplitude de movimento; ANOVA, análise de variância; GRUPO 1, Impacto femoroacetabular e lesão labral; GRUPO 2, Pubalgia e lesão dos adutores; GRUPO 3, Síndrome trocantérica; LL, lado lesionado; LNL, lado não lesionado; RE, rotação externa; RI, rotação interna. 
futebol necessitam de um livre ADM de rotação interna do quadril, seja em flexão ou em extensão. Assim, quando este movimento está limitado, o estresse é aplicado através da articulação para a hemipelve do lado oposto, o que poderia desencadear outros sintomas, como a pubalgia.

Apesar de uma limitação de ADM do quadril não ser descrita como um fator de risco para a síndrome trocantérica, os autores esperavam encontrar algum tipo de alteração neste grupo, visto que, dentro desta síndrome, a maior causa de dor lateral do quadril envolve uma alteração (tendinopatia) dos músculos glúteo médio e/ou mínimo, ${ }^{19}$ que além de serem os principais abdutores do quadril, também são considerados rotadores dessa articulação.

No grupo com diagnóstico de IFA e lesão labral, também era esperada a presença de alterações da amplitude de movimento da articulação coxofemoral, já que o contato inapropriado entre as estruturas articulares poderia ser um limitador da mobilidade ou, por outra análise, poderiam apresentar uma amplitude maior, o que causaria o impacto. Mesmo nos atletas com sintomas unilaterais, não foi observada diferença significativa quando comparado ao lado não lesado. Nos casos de lesão labral, a maioria dos indivíduos apresenta sinais de impacto como descrito por Burnett et $\mathrm{al}^{9}$ sendo esse impacto o responsável pelas lesões. Entretanto, lesões labrais também podem ocorrer por um movimento excessivo de rotação externa do quadril, devido a uma instabilidade atraumática do quadril, com ou sem impacto mecânico.

Uma instabilidade rotacional focal é definida por uma frouxidão localizada de certas estruturas capsuloligamentares que resultam de forças repetitivas de rotação do quadril. ${ }^{20}$ Por isso, a hipótese era a de que haveria alguma alteração na amplitude desses indivíduos, especialmente na ADM de rotação externa, o que não foi observado. ${ }^{20}$

Como mencionado anteriormente, os resultados encontrados no presente estudo não condizem com alguns trabalhos na literatura, que sugerem uma relação entre a limitação de ADM do quadril e lesões desta articulação. O presente trabalho apresenta limitações que podem ter influenciado nos resultados. A primeira limitação encontra-se na avaliação dos atletas, pois apesar da padronização da posição de avaliação adotada pela equipe de fisioterapia (paciente sentado para rotações e decúbito dorsal e ventral para flexão e extensão, respectivamente), os avaliadores eram diferentes, o que pode levar a variações das medidas. Além disso, outra importante consideração a ser feita com relação ao grupo IFA e lesão labral é que o contato entre as estruturas normalmente ocorre em flexão, rotação interna e adução do quadril, e a avaliação foi feita com o paciente sentado, com o quadril em posição neutra em relação a abdução/adução, o que não condiz com a patologia. A segunda limitação do trabalho é a heterogeneidade da amostra, que continha atletas de gêneros, idades e modalidades esportivas diferentes, sendo que esses dados influenciam na ADM do quadril como mostrado por Roach et al. ${ }^{21}$ Essas variantes são tão importantes que há trabalhos demonstrando resultados consistentes a respeito de diferenças do ADM do quadril de jogadores de beisebol. Picha et $\mathrm{al}^{22}$ evidenciaram que jogadores de beisebol entre 7 e 11 anos de idade têm maior rotação interna que aqueles entre 12 e 18 anos de idade. De modo geral, a perna principal tinha mais rotação interna do que a perna de apoio. No entanto, nenhuma diferença de rotação externa do quadril foi observada entre grupos etários, posições dos jogadores ou lado do quadril. Esses achados podem ser explicados pelo aumento da mobilidade e frouxidão ligamentar na faixa etária mais jovem, porque essa população tende a ter mais tecido elástico do que a população adulta.

Outro estudo ${ }^{23}$ avaliou o ADM da articulação coxofemoral como fator de risco de lesão para quadril, abdômen e virilha em jogadores profissionais de beisebol. Eles encontraram correlações entre a diminuição da rotação interna do quadril e o arco total de movimento com lesões isquiotibiais. Ao avaliar o quadril de acordo com a posição do jogador, eles descobriram que os apanhadores e arremessadores tinham arcos de movimento do quadril significativamente diminuídos quando comparados aos jogadores de posição (campo).

É possível notar divergência de resultados entre os estudos devido às diferenças inerentes a cada esporte, idade, gênero e posição do atleta. Mosler et $\mathrm{al}^{24}$ examinaram os perfis de força do quadril e medidas de amplitude de movimento em 394 jogadores de futebol profissional masculinos, assintomáticos. Semelhante ao presente estudo, eles não observaram diferenças clinicamente relevantes entre a perna dominante e não dominante para essas medidas.

Em relação à diferença nos resultados em relação ao gênero, Cheatham et al $^{25}$ avaliaram a amplitude de movimento do quadril em participantes recreativos de treinamento com pesos. Eles observaram que, ao comparar os valores da ADM do quadril entre os gêneros, os homens não apresentaram diferença significativa entre o quadril direito e esquerdo em todos os movimentos. Por outro lado, as mulheres tiveram uma diferença significativa entre o quadril direito e esquerdo para todos os movimentos. Ao comparar os valores de amplitude da coxofemoral entre homens e mulheres, os homens apresentaram menores valores de ADM.

Embora não tenhamos considerado a modalidade praticada pelos indivíduos, Kouyoumdjian et al $^{26}$ mostraram que as atividades que incluem o giro não influenciam na alteração da rotação do quadril. Além disso, a influência da modalidade praticada é de difícil interpretação em uma população em que a maioria é de atletas amadores.

Embora nosso trabalho não mostre diferença na ADM do quadril em relação ao lado lesionado e não lesionado, esse tipo de alteração não deve ser excluído como fator de risco para lesões do quadril. Um estudo de Cibulka et al ${ }^{15}$ mostrou que houve redução da força dos músculos rotadores do quadril quando o indivíduo apresentou diferença entre rotação externa e interna do membro lesionado $\geq 15^{\circ}$. Essa fraqueza causada por alterações no ADM associadas a atividades que exigem estabilidade e potência da articulação do quadril, como pivôs e acelerações/desacelerações abruptas, pode constituir um fator de risco para as estruturas envolvidas nessa articulação.

Verrall et al, ${ }^{27}$ em um estudo prospectivo, também observaram que a assimetria do ADM femoroacetabular precede 0 aparecimento de dor inguinal crônica e pode ser considerada um fator de risco para essa condição. 
Os resultados do Harris Hip Score também não indicaram alteração funcional significativa entre os grupos e não foi possível avaliar se um tipo de lesão foi mais incapacitante que outro. Uma consideração para este resultado é que não houve classificação ou graduação das lesões intragrupo no diagnóstico médico, desconsiderando-se a extensão e gravidade das lesões apresentadas, dificultando sua comparação.

\section{Conclusão}

O presente trabalho não encontrou diferenças no ADM entre as diversas patologias do quadril. No entanto, não devemos excluir essa relação como possível fator de risco para o surgimento de lesões devido às limitações do presente estudo. Essas limitações devem ser corrigidas em estudos futuros.

Conflito de Interesses

Os autores declaram não haver conflito de interesses.

\section{Referências}

1 Feeley BT, Powell JW, Muller MS, Barnes RP, Warren RF, Kelly BT. Hip injuries and labral tears in the national football league. Am J Sports Med 2008;36(11):2187-2195

2 Ansede G, English B, Healy JC. Groin pain: clinical assessment and the role of MR imaging. Semin Musculoskelet Radiol 2011;15(01):3-13

3 Franklyn-Miller A, Richter C, King E, et al. Athletic groin pain (part 2): a prospective cohort study on the biomechanical evaluation of change of direction identifies three clusters of movement patterns. Br J Sports Med 2017;51(05):460-468

4 Morelli V, Weaver V. Groin injuries and groin pain in athletes: part 1. Prim Care 2005;32(01):163-183

5 Alomar AZ. Groin pain in athletes: differential diagnosis, assessment, and management. Saudi J Sports Med. 2015;15(01):3-8

6 Werner J, Hägglund M, Waldén M, Ekstrand J. UEFA injury study: a prospective study of hip and groin injuries in professional football over seven consecutive seasons. Br J Sports Med 2009;43(13):1036-1040

7 Benazzo F, Mosconi M, Viola E. Groin pain in athletes. Sports Med Arthrosc Rev 2000;8:80-85

8 Narvani AA, Tsiridis E, Kendall S, Chaudhuri R, Thomas P. A preliminary report on prevalence of acetabular labrum tears in sports patients with groin pain. Knee Surg Sports Traumatol Arthrosc 2003;11(06):403-408

9 Burnett RS, Della Rocca GJ, Prather H, Curry M, Maloney WJ, Clohisy JC. Clinical presentation of patients with tears of the acetabular labrum. J Bone Joint Surg Am 2006;88(07):1448-1457

10 Anderson K, Strickland SM, Warren R. Hip and groin injuries in athletes. Am J Sports Med 2001;29(04):521-533
11 Ellenbecker TS, Ellenbecker GA, Roetert EP, Silva RT, Keuter G, Sperling F. Descriptive profile of hip rotation range of motion in elite tennis players and professional baseball pitchers. Am J Sports Med 2007;35(08):1371-1376

12 Ibrahim A, Murrell GA, Knapman P. Adductor strain and hip range of movement in male professional soccer players. J Orthop Surg (Hong Kong) 2007;15(01):46-49

13 Tak I, Engelaar L, Gouttebarge V, et al. Is lower hip range of motion a risk factor for groin pain in athletes? A systematic review with clinical applications. Br J Sports Med 2017;51(22):1611-1621

14 Arnason A, Sigurdsson SB, Gudmundsson A, Holme I, Engebretsen L, Bahr R. Risk factors for injuries in football. Am J Sports Med 2004;32(1, Suppl)5S-16S

15 Cibulka MT, Strube MJ, Meier D, et al. Symmetrical and asymmetrical hip rotation and its relationship to hip rotator muscle strength. Clin Biomech (Bristol, Avon) 2010;25(01):56-62

16 Fricker PA, Taunton JE, Ammann W. Osteitis pubis in athletes. Infection, inflammation or injury? Sports Med 1991;12(04):266-279

17 Kendall FP, McCreary EK, Provance PG. Muscles, testing and function: With posture and pain. Baltimore: Williams \& Wilkins; 1993

18 Williams JG. Limitation of hip joint movement as a factor in traumatic osteitis pubis. Br J Sports Med 1978;12(03):129-133

19 Bird PA, Oakley SP, Shnier R, Kirkham BW. Prospective evaluation of magnetic resonance imaging and physical examination findings in patients with greater trochanteric pain syndrome. Arthritis Rheum 2001;44(09):2138-2145

20 Martin RL, Enseki KR, Draovitch P, Trapuzzano T, Philippon MJ. Acetabular labral tears of the hip: examination and diagnostic challenges. J Orthop Sports Phys Ther 2006;36(07):503-515

21 Roach KE, Miles TP. Normal hip and knee active range of motion: the relationship to age. Phys Ther 1991;71(09):656-665

22 Picha KJ, Harding JL, Bliven KC. Glenohumeral and Hip Range-ofMotion and Strength Measures in Youth Baseball Athletes. J Athl Train 2016;51(06):466-473

$23 \mathrm{Li} \mathrm{X}$, Ma R, Zhou H, et al. Evaluation of Hip Internal and External Rotation Range of Motion as an Injury Risk Factor for Hip, Abdominal and Groin Injuries in Professional Baseball Players. Orthop Rev (Pavia) 2015;7(04):6142

24 Mosler AB, Crossley KM, Thorborg K, et al. Hip strength and range of motion: Normal values from a professional football league. J Sci Med Sport 2017;20(04):339-343

25 Cheatham S, Hanney WJ, Kolber MJ. Hip range of motion in recreational weight training participants: a descriptive report. Int J Sports Phys Ther 2017;12(05):764-773

26 Kouyoumdjian P, Coulomb R, Sanchez T, Asencio G. Clinical evaluation of hip joint rotation range of motion in adults. Orthop Traumatol Surg Res 2012;98(01):17-23

27 Verrall GM, Slavotinek JP, Barnes PG, Esterman A, Oakeshott RD, Spriggins AJ. Hip joint range of motion restriction precedes athletic chronic groin injury. J Sci Med Sport 2007;10(06):463-466 